been identified as possible associates of Př́ibram ${ }^{11,12}$. In summer 2000, 4486 Mithra was well placed for observations by radar from Arecibo and Goldstone. The object was found to be in unusual spin state, double-lobed, and more severely bifurcated than any other near-Earth asteroid targeted previously ${ }^{17}$. Such objects are typically believed to be 'rubble piles', loosely bound by gravitation. During close encounters with planets, these objects would be exposed to tidal forces that could lead to their disruption or to the detachment of significant amounts of near-surface rubble that would then be found in orbits similar to that of the parent. This model could possibly resolve the paradox of young streams containing different classes of objects of long cosmic-ray exposure.

Further discussions of the origin of the 'Př́bram stream' must await the final results of the laboratory analyses of Neuschwanstein; more data could be gained from clear identification of other members of this stream (either meteorites or asteroids) in the future. The Neuschwanstein and Př́bram meteorites bring new information to the discussions of the mechanisms of meteorite delivery to the Earth, and demonstrate the importance of long-term fireball observing programmes.

Received 23 January; accepted 28 March 2003; doi:10.1038/nature01592.

1. Ceplecha, Z. Multiple fall of Př́bram meteorites photographed. Bull. Astron. Inst. Czech. 12, 21-47 (1961).

2. Bischoff, A. \& Zipfel, J. Mineralogy of the Neuschwanstein (EL6) chondrite-first results. Lunar Planet. Sci. Conf. XXXIV, abstr. 1212 (2003).

3. Ceplecha, Z. Geometric, dynamic, orbital and photometric data on meteoroids from photographic fireball networks. Bull. Astron. Inst. Czech. 38, 222-234 (1987).

4. Borovička, J., Spurný, P. \& Keclíková, J. A new positional astrometric method for all-sky cameras. Astron. Astrophys. Suppl. Ser. 112, 173-178 (1995).

5. Ceplecha, Z., Spurný, P., Borovička, J. \& Keclíková, J. Atmospheric fragmentation of meteoroids. Astron. Astrophys. 279, 615-626 (1993).

6. Borovička, J., Spurný, P. \& Ceplecha, Z. The Morávka meteorite fall: Fireball trajectory, orbit and fragmentation from video records. Meteorit. Planet. Sci. Suppl. 36, A25-A26 (2001).

Drummond, J. D. A test of comet and meteor associations. Icarus 45, 545-553 (1981).

8. Halliday, I., Griffin, A. A. \& Blackwell, A. T. in Highlights of Astronomy Vol. 6 (ed. West, R. M.) 399-404 (D. Reidel, Dordrecht, 1983).

9. Halliday, I. Detection of a meteorite "stream": Observations of a second meteorite fall from the orbit of the Innisfree chondrite. Icarus 69, 550-556 (1987).

10. Halliday, I., Blackwell, A. T. \& Griffin, A. A. Evidence for the existence of groups of meteoriteproducing asteroidal fragments. Meteorit. Planet. Sci. 25, $93-99$ (1990).

11. Drummond, J. D. Earth-approaching asteroid streams. Icarus 89, 14-25 (1991).

12. Drummond, J. D. The D discriminant and near-Earth asteroid streams. Icarus 146, $453-475$ (2000)

13. Dodd, R. T., Wolf, S. F. \& Lipschutz, M. E. A H chondrite stream: Identification and confirmation. J. Geophys. Res. 98, 15105-15118 (1993).

14. Treiman, A. H. An improbable concentration of basaltic meteorite falls (HED and mesosiderite) in the mid-20 ${ }^{\text {th }}$ century. Meteorit. Planet. Sci. 28, 246-252 (1993).

15. Stauffer, H. \& Urey, H. C. Multiple fall of Př́bram meteorites photographed III. Rare gas isotopes in the Velka stone meteorite. Bull. Astron Inst. Czech. 13, 106-109 (1962).

16. Jenniskens, P. et al. Orbits of meteorite producing fireballs. The Glanerbrug-a case study. Astron. Astrophys. 255, 373-376 (1992).

17. Ostro, S. J. et al. Radar observations of asteroid 4486 Mithra. DPS meeting 32 (American Astronomical Society, Pasadena, California, 2000).

18. Ceplecha, Z. Fireballs photographed in central Europe. Bull. Astron. Inst. Czech. 28, 328-340 (1977).

19. Oberst, J. et al. The "European Fireball Network": Current status and future prospects. Meteorit. Planet. Sci. 33, 49-56 (1998).

20. McCrosky, R. E. \& Boeschenstein, H. The Prairie Meteorite Network. Smithson. Astrophys. Obs. Spec. Rep. 173, 1-23 (1965)

21. Halliday, I., Blackwell, A. T. \& Griffin, A. A. The Innisfree meteorite and the Canadian camera network. J. R. Astron. Soc. Can. 72, 15-39 (1978).

22. McCrosky, R. E., Posen, A., Schwartz, G. \& Shao, C.-Y. Lost City meteorite-Its recovery and a comparison with other fireballs. J. Geophys. Res. 76, 4090-4108 (1971).

23. Brown, P. et al. The orbit and atmospheric trajectory of the Peekskill meteorite from video records. Nature 367, 624-626 (1994).

Acknowledgements We thank the local operators at European Fireball Network (EN) stations for their service at the time of the Neuschwanstein fall, and the finder of the meteorite for the positional information. We also thank J. Keclíková for measuring all photographic records, and Z. Ceplecha and J. Borovička for discussions and contributions to analysis software. The German part of the EN is operated by the German Aerospace Center (DLR), Berlin, the Czech part of the EN is operated by the Astronomical Institute of the Academy of Sciences of the Czech Republic, Ondřejov, and the Austrian station is maintained by the Gahberg Observatory.

Competing interests statement The authors declare that they have no competing financial interests.

Correspondence and requests for materials should be addressed to P.S. (spurny@asu.cas.cz).

\section{Controlled vesicle deformation and lysis by single oscillating bubbles}

\author{
Philippe Marmottant \& Sascha Hilgenfeldt
}

Faculty of Applied Physics, University of Twente, PO Box 217, 7500AE Enschede, The Netherlands

The ability of collapsing (cavitating) bubbles to focus and concentrate energy, forces and stresses is at the root of phenomena such as cavitation damage, sonochemistry or sonoluminescence $^{1,2}$. In a biomedical context, ultrasound-driven microbubbles have been used to enhance contrast in ultrasonic images $^{3}$. The observation of bubble-enhanced sonoporation ${ }^{4-6}-$ acoustically induced rupture of membranes-has also opened up intriguing possibilities for the therapeutic application of sonoporation as an alternative to cell-wall permeation techniques such as electroporation ${ }^{7}$ and particle guns ${ }^{8}$. However, these pioneering experiments have not been able to pinpoint the mechanism by which the violently collapsing bubble opens pores or larger holes in membranes. Here we present an experiment in which gentle (linear) bubble oscillations are sufficient to achieve rupture of lipid membranes. In this regime, the bubble dynamics and the ensuing sonoporation can be accurately controlled. The use of microbubbles as focusing agents makes acoustics on the micrometre scale (microacoustics) a viable tool, with possible applications in cell manipulation and cellwall permeation as well as in microfluidic devices.

Most experiments assessing sonoporation have used strong ultrasonic fields giving rise to a multitude of simultaneous phenomena. The micrographs of ref. 4 suggest that the formation of microjets may be involved, a process that is very difficult to control and model $^{9-11}$. Other candidates for damaging agents during bubble collapse involve shockwaves emitted from the bubble ${ }^{12}$ and/or subsequent heating of the cell wall ${ }^{13}$. All of these processes are significant only when the bubble undergoes a fast, inertial collapse. By constrast, some experiments ${ }^{14}$ demonstrated cell transfection with moderate, off-resonance driving which should have resulted in much weaker bubble oscillations. Our experiments use single microbubbles fixed on a substrate, and use far smaller ultrasound driving amplitudes, replacing the nonlinear dynamics of the inertial collapse with gentle, linear oscillations. Linear oscillations can be sufficient to rupture single cells, because the bubbles' response concentrates ultrasonic energy on the microscale, whereas it could conventionally only be focused to about an ultrasonic wavelength (a few centimetres or millimetres).

In our set-up (Fig. 1a), bubbles of about $10-100 \mu \mathrm{m}$ radius are attached by capillary forces to the walls of a quartz cuvette $(10 \mathrm{~mm} \times 10 \mathrm{~mm} \times 40 \mathrm{~mm})$, after they were generated by syringe injection of air. A piezoelectric transducer provides a standing-wave ultrasound field inside the cuvette, which is filled with a suspension of either cells or lipid vesicles. We chose giant unilamellar lipid vesicles ('artificial cells' consisting only of a lipid bilayer membrane) as our primary object of study because of their well-defined mechanical properties ${ }^{15,16}$. Using electroformation ${ }^{17}$, we obtained 1,2-dioleoyl-sn-glycero-3-phosphocholine (DOPC) vesicles with radii of $10-100 \mu \mathrm{m}$. We use fluorescence marking of either the lipid itself or the interior of the vesicles (when grown in a fluorescent dye solution and then transferred to the experiment). A high-speed camera ( $\leq 2,000$ frames per second) connected to an inverted microscope records phase contrast or fluorescence images of the vesicles' reaction to the bubble oscillation. When not using fluorescence, the contrast between bubbles and solution is enhanced by growing the vesicles in a sucrose solution and transferring them to a 
a

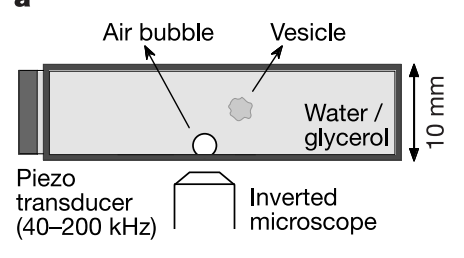

c

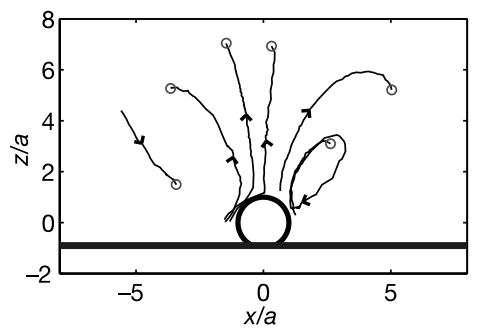

b

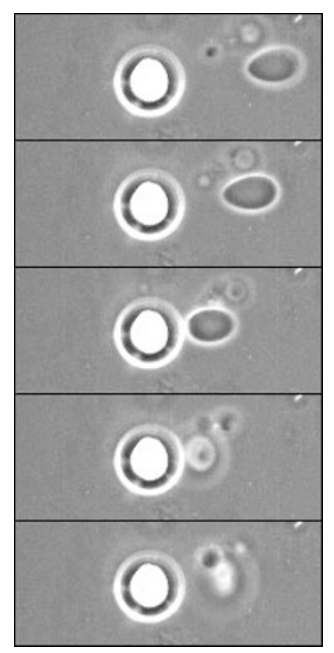

Figure 1 Vesicle motion near an oscillating bubble. a, Layout of the experiment. The piezoelectric transducer generates a standing ultrasound field. b. High-speed time series (bottom view through inverted microscope) of vesicle motion (interframe time $10 \mathrm{~ms}$ ). The bright object is a bubble of radius $a \approx 15 \mu \mathrm{m}$ at the cuvette wall, whose oscillation amplitude is too small to be seen here. The dark object on the right is a lipid vesicle, whose shape far from the bubble would be spherical. Here it is severely deformed as it approaches the bubble, collides with it, and is then expelled away from the observer (upwards in the cuvette), blurring as it leaves the focal plane (see Supplementary movie 1). c, Experimentally observed trajectories of vesicles in a side view ( $z$ is the axis perpendicular to the cuvette wall to which the bubble is attached).

glucose solution of equal osmolarity, but different index of refraction.

Figure $1 \mathrm{~b}$ shows a phase contrast image sequence of a vesicle (radius $R \approx 10 \mu \mathrm{m}$ ) next to a bubble of radius $a \approx 15 \mu \mathrm{m}$ (bright object) attached to the cuvette wall. (See also Supplementary movie 1.) The ultrasound driving at frequency $f=180 \mathrm{kHz}$ is nearresonant for the bubble, but its amplitude is low: the amplitude of bubble oscillation, measured by comparing bubble sizes with and without driving ultrasound, is only $\varepsilon a$, with $\varepsilon \leq 0.05$. From linearized Rayleigh-Plesset bubble dynamics ${ }^{18,19}$, we infer a driving pressure amplitude of about 0.1 bar. Nevertheless, the vesicle reacts with vigorous motion around the bubble (interframe time in Fig. $1 \mathrm{~b}$ is $10 \mathrm{~ms}$ ), being alternately attracted and repelled. Observations with the cuvette tilted by $90^{\circ}$ (now viewing tangentially along the cuvette wall) show that the motion occurs in a plane perpendicular to the wall (Fig. 1c). The vesicles follow loop-like trajectories, approaching the bubble near the wall and then being repelled radially outwards and upwards, away from the wall. The period of this cyclic motion is typically $0.1-1 \mathrm{~s}$, much longer than the ultrasound period. After a few minutes, many vesicles tend to accumulate around the bubble and perform the same motion. They are also deformed in a characteristic fashion: spherical vesicles become prolate when approaching the bubble (see Fig. 1b) and oblate when receding.

When the amplitude of driving is doubled, the speed of the vesicle increases fourfold. This and the observed timescales of the motion suggest that it is a second-order effect of the nonlinearity in the Navier-Stokes equations (rather than an effect of nonlinear bubble dynamics). Modelling can be attempted using the well-established theory of acoustic streaming ${ }^{20}$, where a second-order steady flow emerges from an oscillatory primary flow. Streaming flow from air pockets was suspected in the aggregation and damage of blood platelets and cells around microfilter pores ${ }^{21,22}$. However, the observed recirculation patterns failed to match the then-existing theory ${ }^{23}$ even qualitatively. Elder ${ }^{23}$ explained the streaming of aluminium particles around millmetre-sized bubbles. Lighthill ${ }^{20}$

remarks that in all such engineering applications, the Reynolds number of the steady flow is large ('Stuart streaming'). By contrast, the considerably smaller (micrometre) scales of the present experiments (and of those in refs 21 and 22) lead to a different regime of streaming motion. The bubble-streaming Reynolds number ${ }^{24}$ is:

$$
\operatorname{Re}_{\mathrm{bs}}=\varepsilon^{2}\left(\frac{\omega a^{2}}{\nu}\right)^{1 / 2}
$$

where $\omega=2 \pi f$ is the angular frequency and $\nu \approx 1.0 \times 10^{-6} \mathrm{~m} \mathrm{~s}^{-2}$ is the kinematic viscosity of the aqueous solution. With the values given above, we obtain $\mathrm{Re}_{\mathrm{bs}} \approx 0.04$, much less than in previous microstreaming studies of haemolysis ${ }^{25}$. The secondary flow is therefore Rayleigh-Nyborg-Westervelt (RNW) streaming ${ }^{20}$, which is considerably easier to treat theoretically, as it is an example of Stokes flow.

In our case, the primary oscillation is due to a superposition of bubble volume oscillations and bubble translational oscillations: as the bubble volume changes periodically while its bottom remains fixed to the wall, the centre of mass executes up-and-down motions with amplitude $\varepsilon^{\prime} \approx \varepsilon$ (Fig. 2a). Longuet-Higgins ${ }^{26}$ has calculated the steady RNW streaming flow of such a bubble motion in bulk liquid, resulting in the second-order stream function:

$$
\psi_{2}^{s}=\frac{\varepsilon^{2}}{4} a^{3} \omega \sin (\Delta \phi)\left(-2 \frac{r}{a}+\frac{a}{r}+\frac{a^{4}}{r^{4}}\right) \sin ^{2} \theta
$$

Here, $\Delta \phi$ is the phase shift between volume and translational oscillations, and $r$ the distance to the bubble centre, while $\theta$ is the angle with the axis of translation (that is, gravity). The flow is thus dominated by a finite number of Stokes singularities, in particular a stokeslet $\left(\psi \propto r \sin ^{2} \theta\right)$ and a potential dipole $\left(\psi \propto r^{-1} \sin ^{2} \theta\right)$.

It is now easy to introduce the presence of the cuvette wall using the method of images in Stokes flow ${ }^{27,28}$, where a number of image
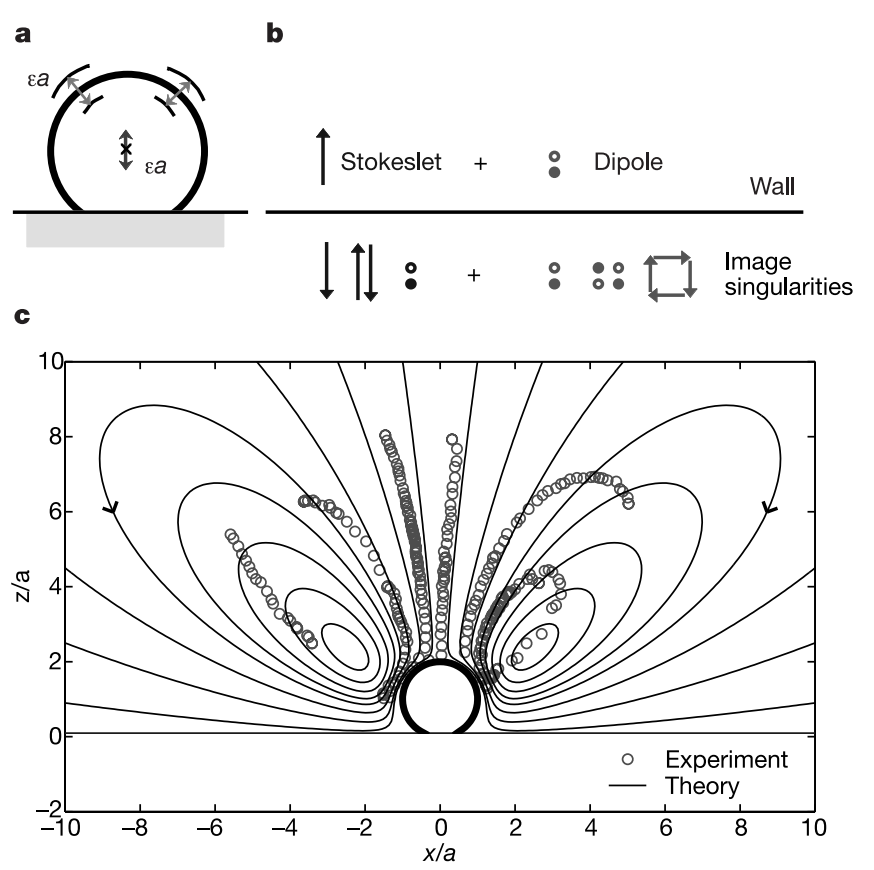

Figure 2 Modelling of the streaming flow. a, A bubble at a wall undergoes both volume and translational oscillations. $\mathbf{b}$, The streaming flow is composed of leading-order stokeslet and dipole singularities. They require three image singularities each to compute the complete flow field in the presence of the wall. c, Streamlines of the computed flow field (for $z>0$ ), superimposed on a number of measured vesicle trajectories, scaled properly. Deviations between experiment and theory show the influence of weak streaming around the vesicles. 
singularities are placed at the opposite side of the wall to make all velocity components at the wall vanish (Fig. $2 b$ ). The Stokes flow fields of these singularities can be simply added up to yield an analytic solution for the flow field. The leading-order far-field term of the complete solution is a dipole-like streaming:

$$
\psi_{2}^{\text {wall }}=-3 \varepsilon^{2} a^{3} \omega \sin (\Delta \phi) \frac{a}{r} \cos ^{2} \theta \sin ^{2} \theta+O\left(\varepsilon^{2} r^{-2}\right)
$$

The streamlines of the complete solution are plotted in Fig. 2c. They are very similar to the vesicle trajectories and illustrate that the vesicles essentially follow the flow as passive tracers. Indeed, as the vesicles (1) have a lipid bilayer in a liquid state, and (2) have a much smaller compressibility modulus than bubbles, the streaming around them should be weaker by up to a factor of $\delta=\left(\nu / \omega a^{2}\right)^{1 / 2}$ compared to the streaming around volume-oscillating bubbles ${ }^{24,26,29}$. For the numbers given above, $\delta \approx 0.06$. The liquid character of the lipid bilayer also leads to tank-treading motion, which is readily observed in our experiments with fluorescence-marked lipid membranes.

The streaming velocities inferred from the complete version of equation (3) are in quantitative agreement with those observed (with a maximum velocity close to the bubble of $u \approx \varepsilon^{2} a \omega \approx 1 \mathrm{~mm} \mathrm{~s}^{-1}$ ). Some vesicles (particularly large ones) seek out the stagnation line of the flow field (at a distance of about $2 a$ from the bubble), where their centre of mass stays stationary while the membrane is undergoing tank-treading and deformation. This situation is particularly well-suited for a controlled change of the forcing parameters to control the degree of deformation of the vesicle. The deformation is determined by the shear rate of the streaming flow field (computed from the rate-ofstrain tensor $\left.G_{i j}=\left(\partial_{i} u_{j}+\partial_{j} u_{i}\right) / 2\right)$ and the vesicle membrane elasticity. For the latter, both thermal fluctuations and material elasticity have to be accounted for ${ }^{16}$.

In the experiments presented so far, the maximum rate of strain as calculated from the theory is $G_{\max } \approx 10^{3} \mathrm{~s}^{-1}$. This leads to a maximum tension in the membrane of $\tau_{\max } \approx \eta G_{\max } R \approx 10^{-4} \mathrm{~N} \mathrm{~m}^{-1}$, where $\eta \approx 10^{-3} \mathrm{~kg} \mathrm{~m}^{-1} \mathrm{~s}^{-1}$ is the dynamic viscosity of the aqueous solution. This tension is sufficient to iron out all the thermal
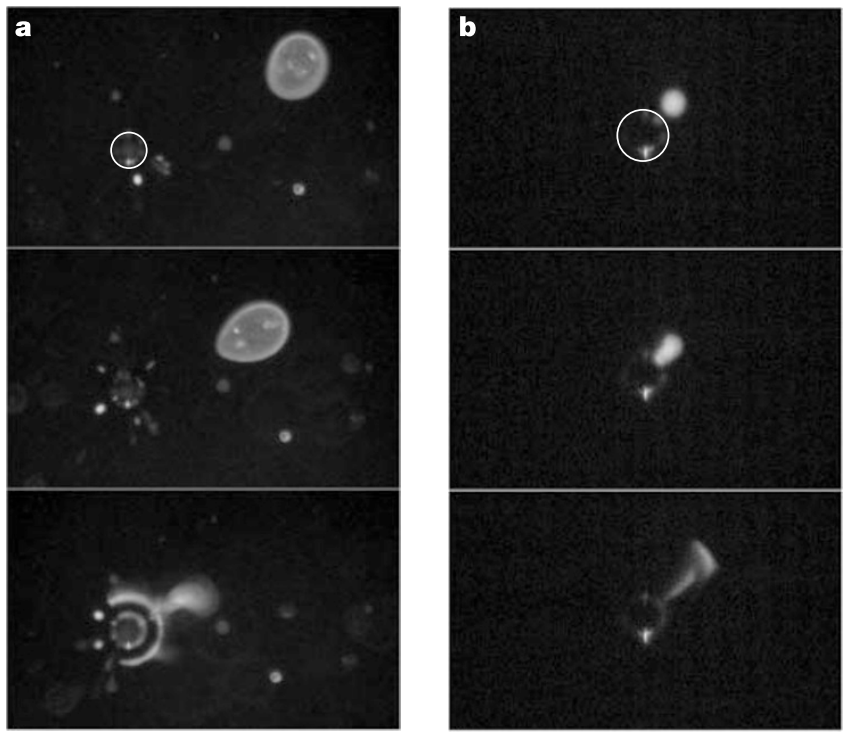

Figure 3 Vesicle rupture. a, A vesicle $(R \approx 40 \mu \mathrm{m})$ with fluorescence-marked lipid membrane approaches a bubble (marked by the white circle, $a \approx 20 \mu \mathrm{m}$ ). The vesicle deforms and fragments. Interframe time is $1 \mathrm{~s}$, ultrasound frequency is $40 \mathrm{kHz}$. b, A tanktreading vesicle $(R \approx 15 \mu \mathrm{m})$ at a stationary position near a bubble (white circle, $a \approx 20 \mu \mathrm{m}$ ). The driving amplitude was increased at about the time of the first frame. The liquid inside the vesicle is fluorescent here, and disperses after vesicle lysis (last frame). Interframe time of $0.4 \mathrm{~s}$, ultrasound frequency $f=180 \mathrm{kHz}$ (see Supplementary movie 2). fluctuations of the membrane area, but will only expand the actual membrane area by a fraction $\Delta A / A \approx 4 \times 10^{-4}$, assuming an area expansion modulus of DOPC of about $0.24 \mathrm{Nm}^{-1}$ (ref. 16) and using the theory of Seifert ${ }^{30}$. To achieve rupture of the membrane (or at least opening of pores), $\triangle A / A$ has to be as large as 0.03 (ref. 16). The present theory therefore predicts that a 100 -fold increase in $\eta G R$ will lead to vesicle rupture.

We tested this prediction by performing the experiment in a water/glycerol mixture with $\eta=20 \eta_{\text {water }}$ and using slightly larger driving amplitudes and vesicle radii. Figure 3 shows two fluorescence imaging series in which rupture can indeed be observed. In Fig. 3a, the vesicle membrane is fluorescence-marked; as the vesicle approaches the bubble, it develops a conical tip in the region of maximum shear rate and then ruptures, with fluorescent debris surrounding the bubble. In Fig. 3b, the liquid inside the vesicle was fluorescence-marked. This vesicle was stationary (tank-treading) close to the bubble when the driving amplitude was increased. The vesicle again shows tip formation and then undergoes complete lysis over an easily controllable timescale of about $1 \mathrm{~s}$. (Also see Supplementary movie 2.) Less dramatic cases of dye leakage have also been observed, which is promising in view of the desired controlled exchange of interior and exterior liquid in drug and DNA delivery applications.

The use of microbubbles as focusing agents makes acoustics a viable tool on the micrometre scale ('microacoustics'). The acoustic streaming flow exerts large enough shear forces on vesicles in the flow to manipulate, deform, and rupture them. With the known parameters from the lipid vesicle experiments, we can now assess the properties of cells, for example, their rupture strain. Preliminary experiments have shown that deformation and lysis of whole HeLa cells is possible in this set-up. Cell viability tests through staining are currently underway. The detailed control over the linear bubble oscillations allows for a quantitative fine-tuning and optimization of this method for cell manipulation, cell wall permeation, or cell lysis. The microstreaming generated by the bubbles offers other interesting perspectives: 'acoustical tweezers' or even microfluidics devices based on this principle become feasible (P. M. and S. H., unpublished work).

Received 27 December 2002; accepted 28 March 2003; doi:10.1038/nature01613.

1. Suslick, K. S. Sonochemistry. Science 247, 1439-1445 (1990).

2. Hilgenfeldt, S., Grossmann, S. \& Lohse, D. A simple explanation of light emission in sonoluminescence. Nature 398, 402-405 (1999).

3. Chang, D. C., Chassey, B. M., Saunders, J. A. \& Sowers, A. E. (eds) Advances in Echo Imaging Using Contrast Enhancement (Kluwer Academic, Dordrecht, 1993).

4. Tachibana, K., Uchida, T., Ogawa, K., Yamashita, N. \& Tamura, K. Induction of cell membrane porosity by ultrasound. Lancet 353, 1409 (1999).

5. Miller, D. L. \& Quddus, J. Sonoporation of monolayer cells by diagnostic ultrasound activation of contrast-agent gas bodies. Ultrasound Med. Biol. 26, 661-667 (2000).

6. Ward, M., Wu, J. \& Chiu, J.-F. Experimental study of the effects of Optison ${ }^{\circledR}$ concentration on sonoporation in vitro. Ultrasound Med. Biol. 26, 1169-1175 (2000).

7. Chang, D. C., Chassey, B. M., Saunders, J. A. \& Sowers, A. E. (eds) Guide to Electroporation and Electrofusion (Academic, New York, 1992).

8. Seki, M., Komeda, Y., Iida, A., Yamada, Y. \& Morikawa, H. Transient expression of beta-glucuronidase in Arabidopsis thaliana leaves and roots and Brassica napus stems using a pneumatic particle gun. Plant Mol. Biol. 17, 259-263 (1991).

9. Prosperetti, A. A new mechanism for sonoluminescence. J. Acoust. Soc. Am. 101, 2003-2007 (1997).

10. Popinet, S. \& Zaleski, S. A front-tracking algorithm for accurate representation of surface tension. Int J. Num. Meth. Fluids 30, 775-793 (1999).

11. Brujan, E.-A., Nahen, K., Schmidt, P. \& Vogel, A. Dynamics of laser-induced cavitation bubbles near an elastic boundary. J. Fluid Mech. 433, 251-281 (2001).

12. Wang, Z. Q., Pecha, R., Gompf, B. \& Eisenmenger, W. Single bubble sonoluminescence: Investigations of the emitted pressure wave with a fiber optic probe hydrophone. Phys. Rev. E59, 1777-1780 (1999).

13. Hilgenfeldt, S., Lohse, D. \& Zomack, M. Sound scattering and localized heat deposition of pulsedriven microbubbles. J. Acoust. Soc. Am. 107, 3530-3539 (2000)

14. Greenleaf, W. J., Bolander, M. E., Sarkar, G., Goldring, M. B. \& Greenleaf, J. F. Artificial cavitation nuclei significantly enhance acoustically induced cell transfection. Ultrasound Med. Biol. 24, 587-595 (1998)

15. Rawicz, W., Olbrich, K., McIntosh, T., Needham, D. \& Evans, E. Effect of chain length and unsaturation on elasticity of lipid bilayers. Biophys. J. 79, 328-339 (2000).

16. Boal, D. Mechanics of the Cell (Cambridge Univ. Press, Cambridge, UK, 2002).

17. Dimitrov, D. S. \& Anguelova, M. I. Lipid swelling and liposome formation on solid surfaces. Prog. Colloid Polym. Sci. 73, 48-56 (1987). 
18. Plesset, M. S. \& Prosperetti, A. Bubble dynamics and cavitation. Annu. Rev. Fluid Mech. 9, 145-185 (1977).

19. Leighton, T. G. The Acoustic Bubble (Academic, London, 1994).

20. Lighthill, J. Acoustic streaming. J. Sound Vib. 61, 391-418 (1978).

21. Miller, D. L., Nyborg, W. L. \& Whitcomb, C. C. Platelet aggregation induced by ultrasound under specialized conditions. Science 205, 505-507 (1979).

22. Miller, D. L. Particle gathering and microstreaming near ultrasonically activated micropores. J. Acoust. Soc. Am. 84, 1378-1387 (1988)

23. Elder, S. A. Cavitation microstreaming. J. Acoust. Soc. Am. 31, 54-64 (1958).

24. Davidson, B. J. \& Riley, N. Cavitation microstreaming. J. Sound Vib. 15, 217-233 (1971).

25. Rooney, J. A. Hemolysis near an ultrasonically pulsating gas bubble. Science 169, 869-871 (1970).

26. Longuet-Higgins, M. S. Viscous streaming from an oscillating spherical bubble. Proc. R. Soc. Lond. A 454, 725-742 (1998).

27. Blake, J. R. \& Chwang, A. T. Fundamental singularities of viscous flow. J. Eng. Math. 8, 23-29 (1974)

28. Pozrikidis, C. Boundary Integral and Singularity Methods for Linearized Viscous Flow (Cambridge Univ. Press, Cambridge, UK, 1992).

29. Riley, N. On a sphere oscillating in a viscous fluid. Q. J. Mech. Appl. Math. 19, 461-472 (1966)

30. Seifert, U. Fluid membranes in hydrodynamic flow fields: Formalism and an application to fluctuating quasispherical vesicles in shear flow. Eur. Phys. J. B 8, 405-415 (1999).

Supplementary Information accompanies the paper on www.nature.com/nature.

Acknowledgements We thank D. Lohse for his support and insight, and A. van den Berg and H. Gardeniers for discussions.

Competing interests statement The authors declare that they have no competing financial interests.

Correspondence and requests for materials should be addressed to P. M.

(p.marmottant@tn.utwente.nl).

\section{Spreading of nanofluids on solids}

\section{Darsh T. Wasan \& Alex D. Nikolov}

Department of Chemical and Environmental Engineering, Illinois Institute of Technology, Chicago, Illinois 60616, USA

Suspensions of nanometre-sized particles (nanofluids) are used in a variety of technological contexts. For example, their spreading and adhesion behaviour on solid surfaces can yield materials with desirable structural and optical properties ${ }^{1}$. Similarly, the spreading behaviour of nanofluids containing surfactant micelles has implications for soil remediation, oily soil removal, lubrication and enhanced oil recovery. But the well-established concepts of spreading and adhesion of simple liquids do not apply to nanofluids ${ }^{2-7}$. Theoretical investigations have suggested that a solid-like ordering of suspended spheres will occur in the confined three-phase contact region at the edge of the spreading fluid, becoming more disordered and fluid-like towards the bulk phase $^{8,9}$. Calculations have also suggested that the pressure arising from such colloidal ordering in the confined region will enhance the spreading behaviour of nanofluids ${ }^{10,11}$. Here we use video microscopy to demonstrate both the two-dimensional crystal-like ordering of charged nanometre-sized polystyrene spheres in water, and the enhanced spreading dynamics of a micellar fluid, at the three-phase contact region. Our findings suggest a new mechanism for oily soil removal-detergency.

When a gas bubble or oil/liquid drop dispersed in an aqueous nanofluid approaches a smooth, horizontal hydrophilic solid surface, there is a microscopic transition between the liquid film and the meniscus-and the nanofluid film can change its thickness in steps $^{11}$. The transition region between the liquid film on a solid surface and the bulk meniscus has a wedge-like profile (Fig. 1), and its shape is determined by forces arising from the ordering of particles or supermolecules, such as surfactant micelles. Structural transitions have been observed in colloidal fluids confined to very thin wedges ${ }^{12-15}$.

The aim of this study is to reveal the effects of the particle structure formation and the structural disjoining pressure on the spreading of colloidal fluids on solid surfaces. Spreading is generally described in terms of the spreading coefficient $S$, given by $S=\sigma_{\mathrm{sg}}-\sigma_{\mathrm{sl}}-\sigma_{\mathrm{lg}}$, where $\sigma$ is the respective interfacial tension existing between the solid-gas $\left(\sigma_{\mathrm{sg}}\right)$, solid-liquid $\left(\sigma_{\mathrm{sl}}\right)$ and liquidgas $\left(\sigma_{\mathrm{lg}}\right)$ phases. The de Gennes theory relates the value of $S$ to the disjoining pressure of a wetting film $(\Pi(h)$, where $h$ is the thickness of the film) $\mathrm{as}^{2,16}$ :

$$
S=\Pi(h) h+\int_{h} \Pi(h) \mathrm{d} h
$$

We directly observed the particle-structuring phenomenon in the liquid film-meniscus region by using reflected-light digital video microscopy. The wedge film was formed by blowing a small air bubble (diameter $200 \mu \mathrm{m}$ ) against an optically smooth glass plate in a suspension of monodisperse, charged latex spheres in deionized surfactant-free water. The volume fraction of spheres in the fluid
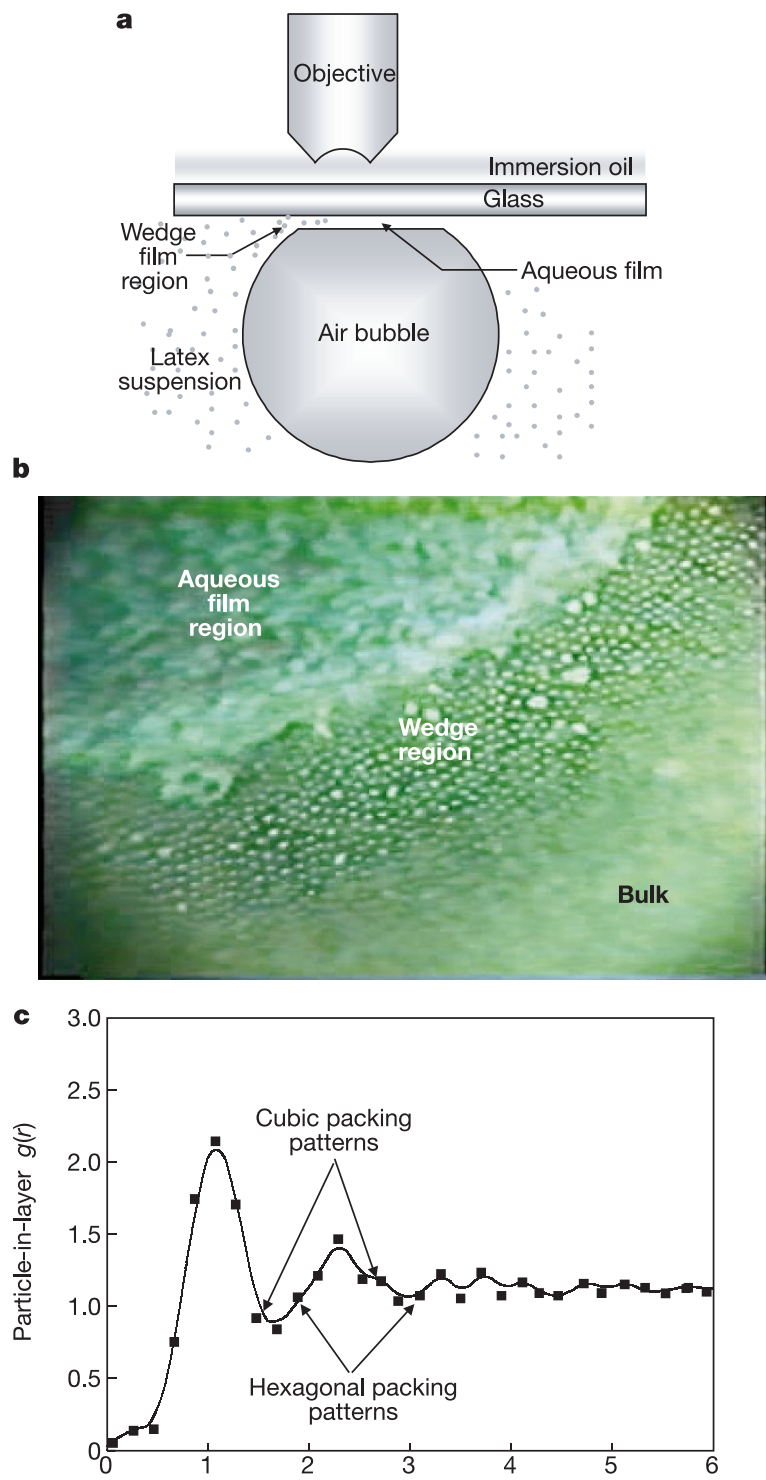

Particle centre-to-centre distance scaled by diameter, $r / d$

Figure 1 Particle structuring in a wedge film. a, Diagram of experimental set-up. b, Particle structuring in a wedge film. Latex particles had diameter $1 \mu \mathrm{m}$, charge $0.8 \mu \mathrm{C} \mathrm{cm}^{-2}$, and occupied 7 vol.\%. c, In-layer particle structure inside the wedge film. Mean particle-to-particle distance was $1.2 d$; effective particle volume was 44 vol.\%. 